\title{
Comportamiento nutricional y perfil alimentario de la producción lechera en pastos cultivados (Panicum maximum Jacq)
}

\author{
Nutritional behavior and feeding profile of dairy production in cultivated pastures \\ (Panicum maximum Jacq)
}

\author{
Jimny Núñez Delgado ${ }^{1,3}$, Javier Ñaupari Vásquez ${ }^{1,2}$, Enrique Flores Mariazza ${ }^{1,2}$
}

\section{Resumien}

\begin{abstract}
Se evaluaron las variables inherentes al componente forrajero y animal para diseñar perfiles alimentarios de vacas de doble propósito sobre pasturas de Panicum maximum Jacq en un fundo ganadero de la selva alta peruana. Las variables forrajeras fueron tasa de crecimiento y disponibilidad de forraje y las variables animales fueron la composición química de la dieta y el perfil alimentario. El experimento se realizó en las épocas seca, inicio de lluvias y lluviosa. Se instalaron 14 excluidores en 41.9 has y se trabajó con cinco vacas adultas Brown Swiss x criollo en ordeño. Las tasas de crecimiento y disponibilidad se obtuvieron mediante el método de corte y separación manual, la dieta se obtuvo por simulación manual, el requerimiento energético a través del presupuesto de actividades y el perfil alimentario mediante el balance entre la demanda y oferta de forraje. Se utilizó un diseño completamente al azar para crecimiento y disponibilidad de forraje, y bloques al azar para la composición química. La tasa de crecimiento de forraje varió entre 8.16 y $46.28 \mathrm{~kg} \mathrm{MS} /$ ha/día; la disponibilidad de forraje entre 2696.9 y $7764.3 \mathrm{~kg} \mathrm{MS} / \mathrm{ha}$ para las épocas seca y lluviosa, respectivamente. La concentración de energía metabolizable en el forraje fue 3.94 y $5.23 \mathrm{EM} \mathrm{MJ} / \mathrm{kg} \mathrm{MS}$ y la demanda total de energía de las vacas fue 102.0 y $90.1 \mathrm{MJ}$ EM/día para las épocas seca y lluviosa, respectivamente. El perfil alimentario reveló un excedente en la época lluviosa y un déficit en las épocas seca e inicio de lluvia. Se concluye que el establecimiento ganadero está subutilizando la pastura, especialmente en la época lluviosa, debido a la buena disponibilidad forrajera y baja carga animal.
\end{abstract}

Palabras clave: perfil alimentario; simulación manual; crecimiento; disponibilidad de forraje; pastura; comportamiento animal

\footnotetext{
${ }^{1}$ Laboratorio de Ecología y Utilización de Pastizales, Universidad Nacional Agraria La Molina, Lima, Perú

${ }^{2}$ Departamento de Producción Animal, Facultad de Zootecnia, Universidad Nacional Agraria La Molina, Lima, Perú

${ }^{3}$ E-mail: jimny_unalm@hotmail.com

Recibido: 11 de mayo de 2018

Aceptado para publicación: 20 de noviembre de 2018
} 
Variables inherent to the forage and animal component were evaluated to design feed profiles of dual-purpose cows grazing Panicum maximum Jacq in a dairy farm in the Peruvian tropics. The forage variables were growth rate and forage availability and the animal variables were the chemical composition of the diet and feed profile. The experiment was carried out in the dry, early rainy and rainy seasons. Fourteen enclosures were installed in 41.9 ha and five Brown Swiss x Creole lactating cows were used. Forage growth and availability rates were obtained through cutting and manual separation, diets were obtained by manual simulation, energy requirement through the budget of activities and feed profile through the balance of forage demand and supply. A completely randomized design was used for forage growth and availability, and random blocks for chemical composition. The forage growth rate varied between 8.16 and $46.28 \mathrm{~kg}$ $\mathrm{DM} / \mathrm{ha}$ /day; forage availability between 2696.9 and $7764.3 \mathrm{~kg} \mathrm{DM} / \mathrm{ha}$ for the dry and rainy seasons, respectively. The concentration of metabolizable energy in the forage was 3.94 and 5.23 ME MJ/kg MS and total energy demand of cows was 102.0 and $90.1 \mathrm{MJ} \mathrm{ME} /$ day for the dry and rainy seasons, respectively. Feed profile revealed a surplus in the rainy season and a deficit in the dry and early rainy seasons. It is concluded that the farm is underutilizing the pasture, especially in the rainy season, due to good forage availability and low stocking rate.

Key words: feed profile; manual simulation; growth; forage availability; pasture; animal behavior

\section{INTRODUCCIÓN}

Los sistemas ganaderos lecheros tropicales son de naturaleza silvopastoril en condiciones de secano, con rendimientos de leche promedio de 4.91 /día dependiendo de los sistemas de manejo (INEI, 2012). La producción de forraje en secano es dependiente de la temporalidad de las precipitaciones. Los planes de alimentación deben basarse en el requerimiento nutricional de los animales y en la disponibilidad de forraje y nutrientes, haciendo un balance entre la producción total de forraje y la demanda de alimento del sistema de producción (Ñaupari y Flores, 2002).

La oferta de forraje comprende la tasa de crecimiento y su disponibilidad. La pobre fertilidad de los suelos tropicales y las grandes variaciones en las precipitaciones pluviales son los principales parámetros limitantes y de mayor importancia en la producción de forraje. La tasa de crecimiento del pasto se incrementa rápidamente después del pastoreo, y luego se hace más lento a medida que la masa vegetal, el área foliar y la intercepción de luz aumentan. El crecimiento de la planta se afecta por la disponibilidad de nutrientes en el suelo (Barreira, 2008) y por la temperatura ambiental, considerándose un óptimo fotosintético entre 30 a $35^{\circ} \mathrm{C}$ y 35 a $39^{\circ} \mathrm{C}$ para leguminosas y gramíneas tropicales, respectivamente (Baruch y Fisher, 1991). Sin embargo, se considera que el aumento de la temperatura en la superficie, producto del cambio climático, afectará el crecimiento de las plantas (Peng et al., 2004), generando estrés por calor, causando alteraciones morfoanatómicas, fisiológicas y bioquímicas (Wahid et al., 2007) y su fenología en general (Snider et al., 2009). 
Se ha determinado que Panicum maximum y especies de Brachiaria extraen 79.0 y $63.0 \mathrm{~kg} / \mathrm{ha} /$ año de nitrógeno, respectivamente (Bernal y Espinoza, 2003), habiendo una gran diferencia entre especies (Cerdas, 2011). A pesar de la capacidad de extracción de nutrientes, el contenido de proteína cruda (PC) de las gramíneas puede variar entre 3\% en una gramínea tropical muy madura hasta más de $30 \%$ en una pastura muy tierna y fertilizada (Molano, 2012), disminuyendo con la edad (Verdecia et al., 2008). Así mismo, el contenido de pared celular está inversamente relacionado con el contenido de proteína, mientras el contenido de celulosa suele ser de 20 a $30 \%$ de la materia seca (MS), en tanto que las hemicelulosas pueden variar entre 10 y $30 \%$. Los carbohidratos forman la tercera o cuarta parte del peso seco de las plantas, representando el 45 a $80 \%$ de la MS, constituyendo la principal fuente de energía para los rumiantes (Molano, 2012).

La digestibilidad es uno de los factores que influencia el consumo de los alimentos por los rumiantes. La digestibilidad de la MS y la materia orgánica (MO) en especies de Panicum disminuyen con la edad de la planta, mostrando su mejor comportamiento a los 30 días de edad con 63.5 y $68.7 \%$ y el más bajo a los 105 días con 49.8 y $51.9 \%$, respectivamente (Verdecia et al., 2008).

La ingesta de forrajes está influenciada por la época del año y el estado fenológico de la planta (Harper y McNeill, 2015; Vidal, 2008). El consumo de nutrientes es el producto de la cantidad de forraje consumido y la concentración de nutrientes en ese forraje y la eficiencia de conversión de nutrientes en producto animal, el cual comprende las eficiencias en los procesos digestivos y metabólicos (Derner et al., 2017).

El cálculo de capacidad de carga de la pastura se basa, generalmente, en la disponibilidad de forraje mensual, sin considerar las tasas de crecimiento del pasto, por lo que este procedimiento es impreciso (Ñaupari y Flores, 2002). La capacidad de carga en unidades animal (U.A.) se calcula dividiendo la producción de forraje derivada del crecimiento entre la demanda anual del sistema. La demanda anual del sistema se refiere a la asignación de forraje en $\mathrm{kg} \mathrm{MS/UA/día,} \mathrm{o} \mathrm{al} \mathrm{re-}$ querimiento de energía, MJ EM/UA/día, siendo este último el más recomendado (Milligan et al., 1987; Naupari y Flores, 2002).

Los perfiles alimentarios constituyen una primera aproximación para balancear la oferta y demanda de forraje, la cual puede ajustarse con el monitoreo del forraje disponible y el rendimiento productivo de los animales (Milligan et al., 1987; Naupari y Flores, 2002). Estos perfiles se usan para estimar la carga potencial y establecer un balance estacional entre la demanda del hato y el patrón esperado de oferta de la pastura, dándole un uso racional al recurso forrajero sin inducir retrogresión. En consecuencia, los perfiles alimentarios se convierten en una herramienta clave para la toma de decisiones en un entorno flexible para la resolución de problemas de los sistemas ganaderos tropicales altamente dependientes de la temporalidad climática.

No existen estudios sobre el manejo estacional del pastoreo y calidad nutricional de Panicum maximum Jacq en praderas tropicales de ceja de selva del Perú que permitan mejorar la eficiencia en la producción y utilización de las praderas para generar indicadores de manejo de fácil adopción. En consecuencia, los objetivos del trabajo fueron determinar las tasas de crecimiento y disponibilidad de forraje de pasturas de Panicum maximum Jacq, estimar el estatus nutricional de vacas al pastoreo a partir de sus requerimientos y calidad de la dieta y diseñar un perfil alimentario del hato lechero típico de la zona para establecer la capacidad de carga que satisface las necesidades de forraje de los animales. 


\section{Materiales y Métodos}

El presente estudio se llevó a cabo durante el periodo 2012-2013 en la Unidad Agropecuaria San Antonio del distrito de Bellavista, provincia de Jaén, Cajamarca (Perú). Es un fundo de mediano tamaño, típica de ceja de selva. Se encuentra a 729 msnm, y la zona está clasificada de acuerdo con Holdridge (2000) como bosque seco premontano tropical (bs-PT). La temperatura media anual es de $24.2^{\circ} \mathrm{C}$ con máximas y mínimas promedio de 34.0 y $18.5^{\circ} \mathrm{C}$, respectivamente y la precipitación anual es 722.6 $\mathrm{mm}$. El fundo tiene un área 54 ha divididos en 19 potreros con cercas fijas, bajo un sistema silvopastoril, donde el componente pasto es Panicum maximum Jacq, el componente arbóreo es Acacia macracantha (árbol nativo, con una densidad promedio de 60 árboles/ha) y el componente animal 25 vacas Brown Swiss x criollo en producción. Se evaluaron 41.9 has divididas en 14 potreros destinados a las vacas en producción, estando el resto del área destinado a la recría y a las vaquillas. Los potreros tenían un periodo de descanso entre 20 y 45 días dependiendo de la época del año.

Se utilizaron cinco vacas Brown Swiss $\mathrm{x}$ criollo con 2 meses de lactación, en las épocas seca (junio - setiembre), inicio de lluvia (octubre - enero) y lluviosa (febrero marzo). Las características fisicoquímicas del suelo reportadas por el Laboratorio de Análisis de Suelos y Aguas de la UNALM fueron suelo de textura franco-arcillo-arenoso, con un $\mathrm{pH}$ ligeramente alcalino (7.77) y bajo nivel de materia orgánica $(1.59 \%)$. El contenido de fósforo es medio (9.00 ppm), pero el contenido de potasio (246.50 ppm) es alto (D.S. $\mathrm{N}^{\circ}$ 017-2009-AG, 2009), siendo próximos a los valores reportados por Romero (2018), bajo contenido de fósforo $(6.35$ ppm) y alto contenido de potasio $(339.25$ ppm) en sistemas silvopastoriles de ceja de selva.

\section{Forraje}

El crecimiento del forraje se midió dentro de 14 excluidores de $4 \mathrm{~m}^{2}$ ubicados en cada potrero. El área de muestreo fue de 1 $\mathrm{m}^{2}$ para eliminar el efecto del borde (ASRM, 1962). La tasa de crecimiento fue el forraje disponible dentro del excluidor un mes después del corte, expresada en kilogramos de forraje seco por hectárea por día ( $\mathrm{kg} \mathrm{MS} / \mathrm{ha} / \mathrm{d})$.

La disponibilidad de pasto ( $\mathrm{kg} \mathrm{MS} / \mathrm{ha}$ ) se midió utilizando el método de corte y separación manual por especies y partes (ASRM, 1962), el cual consistió en cortar el material forrajero a $10 \mathrm{~cm}$ del suelo, separando el material senescente y partículas de suelo del forraje. La disponibilidad de forraje se midió en los 14 potreros utilizando un cuadrante de fierro de $1 \mathrm{~m}^{2}$, previo a la entrada de los animales. En ambos casos se utilizó la balanza digital (OHAUS ${ }^{\circledR}$, Ranger 3000) con capacidad de $3000 \mathrm{~g}$ y precisión de $0.1 \mathrm{~g}$.

La temperatura del suelo se midió dentro de un radio de $2 \mathrm{~m}$ a las jaulas de crecimiento del pasto, a una profundidad de $15 \mathrm{~cm}$ de la superficie, mediante un geotermómetro por $5 \mathrm{~min}$. Las lecturas fueron entre las 09:00 y 12:00 horas. La humedad del suelo se midió por gravimetría (Martínez, 1971). El muestreo se realizó entre 10 y $20 \mathrm{~cm}$ de profundidad, utilizando envases con cierre hermético para evitar la pérdida de humedad. Las muestras se colocaron en estufa a $105^{\circ} \mathrm{C} / 24 \mathrm{~h}$.

\section{Valor Nutritivo de la Dieta}

Por un periodo de una semana en cada época de evaluación y en siete potreros representativos se observó el pastoreo individual de los animales por 2-3 h, para luego tomar muestras manuales representativas del forraje consumido por el animal por el método de simulación manual en 100 estaciones alimentarias (Flores, 1993). Se obtuvo una alícuota de $1 \mathrm{~kg}$ para el análisis en el laboratorio. 
La digestibilidad in vitro de la materia orgánica (DIVMO) se analizó por el método de Tilley y Terry (1963). Luego se determinó el Valor Energético del Forraje (VEF) en términos de energía metabolizable (MJ EM/Kg MS) mediante: $\mathrm{EM}=0.16 \times$ DIVMO (MAFF, 1975; Geenty y Rattray, 1987). El contenido de proteína cruda, fibra detergente neutra, calcio y fósforo se determinó empleando el método semi-micro Kjeldahl, análisis de fibra de forrajes, titulación con permanganato de potasio, y espectrofotometría con molibdato de amonio, respectivamente (AOAC, 2001).

\section{Diseño de Perfiles Alimentarios}

El perfil alimentario consistió en hacer un balance entre la oferta y demanda de forraje para el periodo de estudio (Milligan et al., 1987; Ñaupari y Flores, 2002). La oferta de forraje se estimó a partir de la tasa de crecimiento y producción de forraje, y la demanda a partir de los requerimientos energéticos de una vaca y la capacidad de carga. La producción promedio anual (kg MS/ha/ año) de pasto se estimó a partir de la integral de las tasas de crecimiento de forraje mensual (kg MS/ha). La tasa de crecimiento mensual se obtuvo del crecimiento diario multiplicado por el número de días correspondientes al mes.

La cantidad de leche promedio producida para la época fue registrada en cinco vacas luego de cada ordeño y en cada época. La producción promedio diaria de grasa en la leche (kg grasa/vaca/día) fue obtenida multiplicando la producción de leche promedio diaria por el porcentaje de grasa. El porcentaje de grasa se obtuvo por el método Gerber (Alais, 1994). Los requerimientos de energía metabolizable, MJ EM/vaca/día y el valor nutritivo de la pastura expresado en $\mathrm{MJ}$ EM/kg MS de forraje se calcularon para las épocas de estudio. Los requerimientos energéticos (MJ EM/vaca/día) se determinaron por comportamiento animal, en base al tiempo invertido en cada actividad; con base a los requerimientos de mantenimiento más actividad al pastoreo y a los requerimientos de producción de leche (Geenty y Rattary, 1987; NRC, 2001). Este método consistió en hacer un seguimiento por $42 \mathrm{~h}$ seguidas a cada animal en la que se registra cada $5 \mathrm{~min}$ la actividad que realiza; luego se utilizaron las siguientes ecuaciones: mantenimiento $(550 \mathrm{kj} /$ $\left.\mathrm{kg} \mathrm{W}^{0.75}\right)$, pastoreo, caminata y parada (2.3, 1.0 y $0.5 \mathrm{kj} / \mathrm{hora} / \mathrm{kg}$ PV, respectivamente), caminata (2.5 kj/kilómetro $\mathrm{PV}$ ) y producción de leche $(5100 \mathrm{kj} / \mathrm{kg}$ leche). La asignación diaria de pasto por vaca ( $\mathrm{kg} \mathrm{MS/vaca/día)} \mathrm{para}$ cubrir sus requerimientos de energía se obtuvo al dividir los requerimientos de energía (MJ EM/vaca/día) entre el valor energético del forraje VEF (MJ EM/kg MS).

La demanda de forraje se obtuvo sumando las asignaciones en cada periodo de evaluación de una vaca multiplicada por el número de días correspondientes al periodo. La carga animal propuesta fue asignada constante y la demanda de pasto fue expresada en kg MS/ha/día, multiplicando la asignación diaria de forraje de una vaca por la capacidad de carga propuesta.

El cambio en la disponibilidad resultó de restar la demanda del hato al crecimiento de forraje, multiplicado por el número de días correspondiente en la época. La disponibilidad al inicio de época se refirió a la producción de forraje encontrada en la época y la disponibilidad más crecimiento estuvo referida a toda la época. La disponibilidad al final de la época se calculó con la siguiente fórmula y expresada en porcentaje: [(Disponibilidad más crecimiento) x (Disponibilidad al final del mes) / (Disponibilidad más crecimiento) $] * 100$.

\section{Análisis Estadístico}

Los resultados experimentales se evaluaron utilizando estadística descriptiva y análisis de variancia. La variación de la tasa de crecimiento y los valores de disponibilidad de forraje fueron analizados bajo un diseño completamente al azar utilizando las épocas como tratamientos y los excluidores de crecimiento como réplicas. El análisis de varianza para 
las actividades en general se realizó utilizando un diseño jerárquico, donde los tratamientos fueron las épocas y las variaciones entre vacas dentro de las épocas como error experimental, y las variaciones entre los días dentro de las vacas como error de muestreo. Para la evaluación de comparaciones individuales de los efectos se utilizó la prueba de medias de Duncan, utilizando un nivel de 0.05 para detectar diferencias estadísticas entre promedios.

\section{Resultados y Discusión}

\section{Crecimiento y Disponibilidad de Forraje}

La tasa de crecimiento fue significativamente superior $(\mathrm{p}<0.05)$ en la época lluviosa (46.28 kg MS/ha/día), seguido del inicio de lluvias (18.42 kg MS/ha/día) y de la época seca (8.16 kg MS/ha/día) (Figura 1).

Las tasas de crecimiento en las tres épocas fueron similares a los valores de 15.9 y $65.7 \mathrm{~kg} \mathrm{MS} / \mathrm{ha} /$ día cortado a las tres semanas, en épocas seca y lluviosa, respectivamente, reportados por Ramírez et al. (2009) en $P$. maximum, Por su parte, Verdecia et al. (2008) encontraron tasas de crecimiento acumulado de 47.7 y $120.7 \mathrm{~kg} \mathrm{MS} / \mathrm{ha} /$ día en condiciones bajo riego durante las épocas seca y lluviosa, respectivamente, en P. maximum var. Mombaza.

Las mayores tasas de crecimiento en la época lluviosa se debieron a la mayor humedad $(18.0 \%)$ y temperatura del suelo $\left(24.7^{\circ} \mathrm{C}\right)$, radiación solar y la cantidad de nutrientes en el suelo que en conjunto intervienen en el crecimiento y procesos fenológicos de la planta (Zaragoza et al., 2009). La producción de forraje es directamente proporcional a la humedad del suelo (López et al., 2018). La mayoría de las especies forrajeras tropicales tiene un sistema fotosintético C4, dándole la propiedad de no saturarse ni con la máxima radiación solar debido a su alta tasa fotosintética y ausencia de fotorrespiración, independientemente del rendimiento cuántico máximo de captación del $\mathrm{CO}_{2}$, incluso por debajo de $25^{\circ} \mathrm{C}$ (Long et al., 2006). Las especies de Panicum pueden soportar la sombra sin ser foto-inhibidas, mostrando un potencial asociativo con los árboles (Pentón, 2000).

La disponibilidad de forraje fue mayor en la época lluviosa (7764.3 kg MS/ha) debido a las mejores condiciones de temperatura $\left(24.7^{\circ} \mathrm{C}\right)$, humedad del suelo $(18.0 \%)$ y precipitación $(982.0 \mathrm{~mm})$ que favorecieron el crecimiento. La sobreproducción en la época lluviosa genera la acumulación de forraje residual debido a la subutilización, resultando una mayor proporción de forraje maduro, lo cual disminuye la calidad nutritiva de la pastura debido a la acumulación de carbohidratos en la parte basal de los tallos a 7-10 cm del suelo (Bernal y Espinoza, 2003). Cerdas y Vallejos (2011) y Ramírez et al. (2009) encontraron tendencias similares de mayor disponibilidad de $P$. maximum en la época lluviosa.

\section{Valor Nutritivo de la Dieta}

\section{Proteina cruda}

El contenido promedio de proteína cruda (PC) fue estadísticamente diferente entre épocas $(\mathrm{p}<0.05)$. La mayor cantidad de PC se encontró al inicio de la época de lluvias (16.64\%), seguida de la época lluviosa $(13.76 \%)$ y de la época seca (10.49\%) (Figura 2). Este comportamiento se debe que al inicio de las lluvias existen mayor cantidad de rebrotes de hojas y tallos suaves y palatables, mientras que en la época lluviosa se encontraron estructuras más gruesas y lignificadas debido a la floración y maduración del forraje, y en la época seca el forraje se encuentra totalmente maduro y lignificado. Ruiz et al. (2015) reporta que el contenido de proteína cruda varía entre $18.70,14.20$ y $11.30 \%$ a los 30,60 y 90 días de rebrote, respectivamente; mientras que Baldelomar et al. (2004) reportaron 14 y $11 \%$ de PC a los 20 y 100 días de corte, respectivamente. 


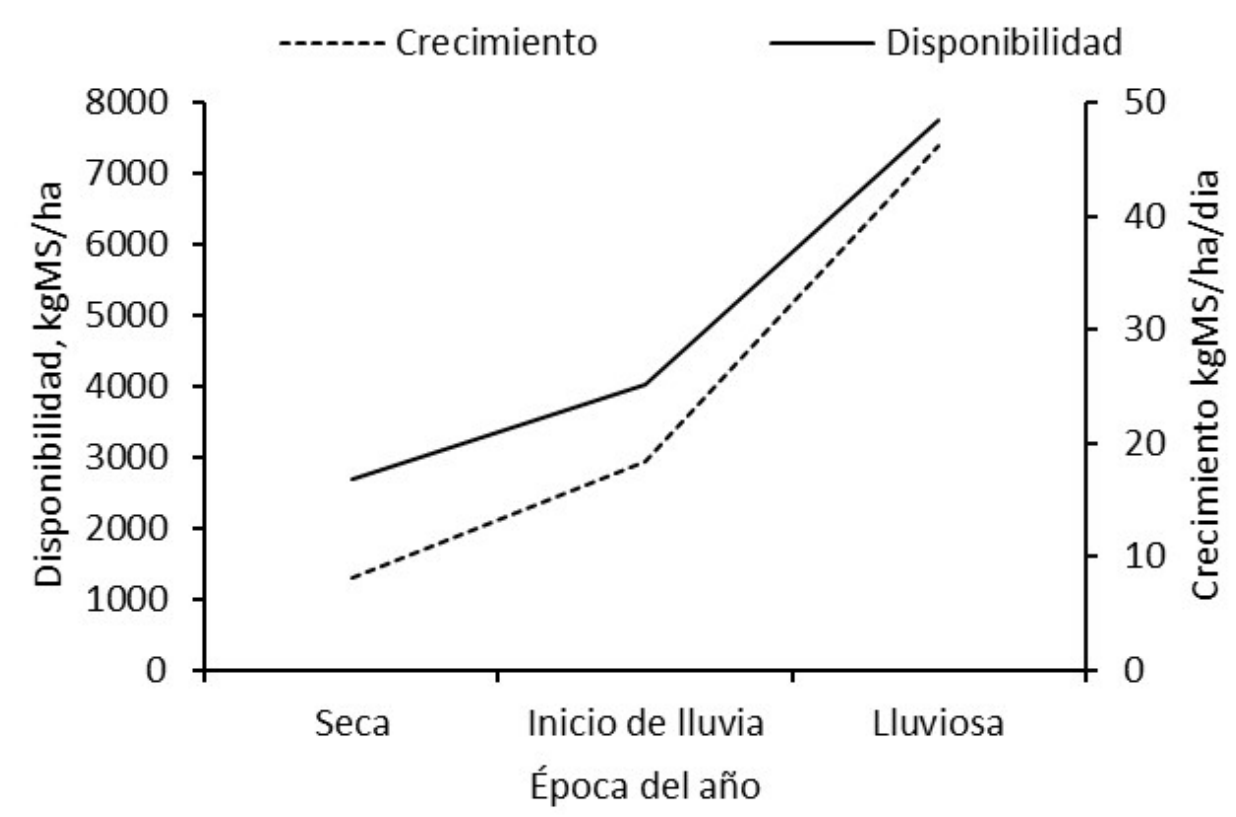

Figura 1. Tasa de crecimiento ( $\mathrm{kg} \mathrm{MS} / \mathrm{ha} /$ día) y disponibilidad de forraje (Kg MS/ha) del pasto Panicum maximum Jacq, según época del año

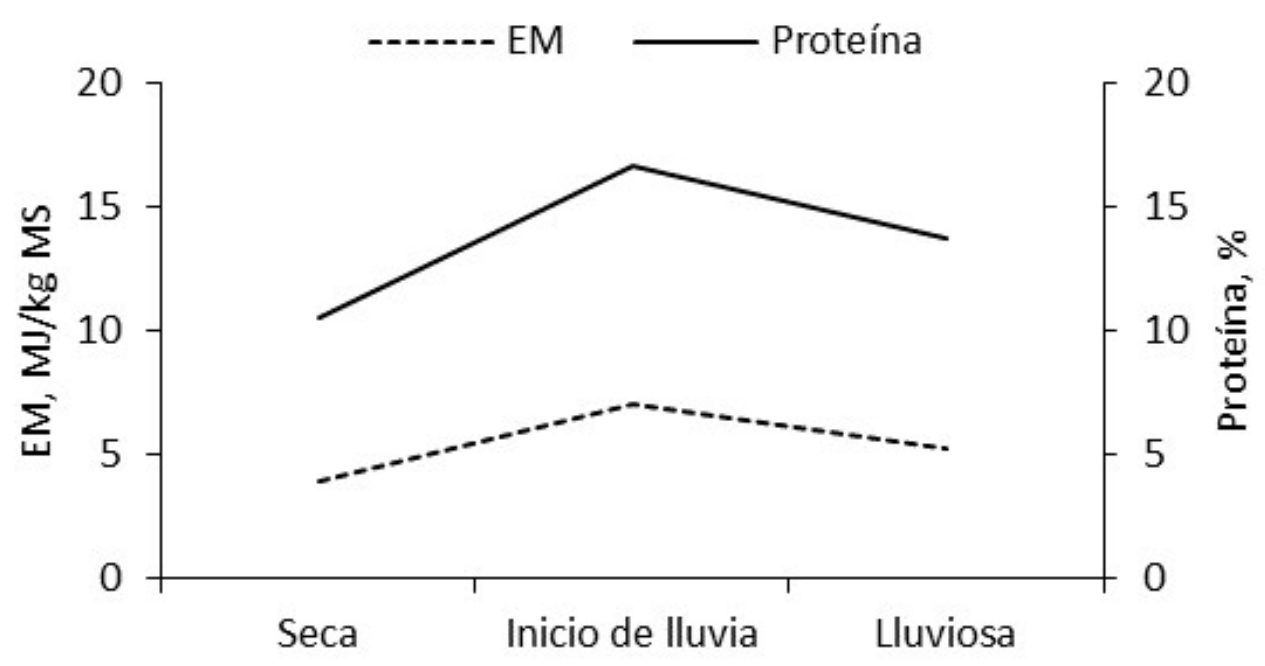

Figura 2. Niveles de proteína cruda (\%) y energía metabolizable (MJ/kg MS) en la dieta de vacas Brown Swiss x criollo alimentadas al pastoreo sobre una pastura de Paniucum maximum en Cajamarca, Perú 


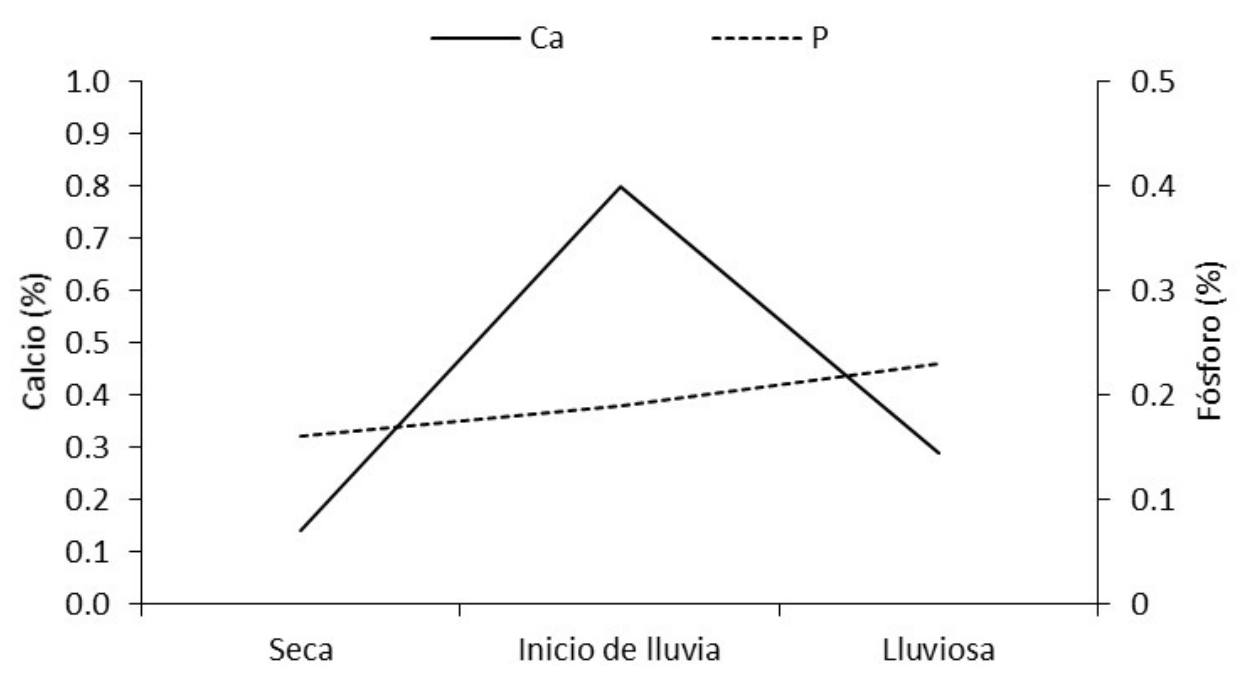

Figura 3. Niveles (\%) de calcio y fósforo en la dieta de vacas Brown Swiss x criollo alimentadas al pastoreo sobre una pastura de Paniucum maximum en Cajamarca, Perú

\section{Energía metabolizable}

La energía metabolizable (EM) fue significativamente diferente entre épocas $(\mathrm{p}<0.05): 3.94,7.00$ y $5.23 \mathrm{MJ} \mathrm{EM} / \mathrm{kg} \mathrm{MS}$ en épocas seca, inicio de lluvia y lluviosa, respectivamente (Figura 2). Similares resultados fueron reportados por Verdecia (2008) en $P$. maximum $(7.45$ y $7.61 \mathrm{MJ} / \mathrm{kg}$ MS en periodo lluvioso y poco lluvioso, respectivamente, a los 105 días de rebrote); sin embargo, la máxima EM reportada por este autor fue de $10.17 \mathrm{MJ} / \mathrm{kg} \mathrm{MS}$ a los 30 días de edad. El menor valor de EM en la época seca se debe a la mayor concentración de tejido fibroso. Asimismo, el valor energético del forraje disminuye con la edad, debido a una menor concentración de carbohidratos solubles, proteína digestible y digestibilidad de la materia orgánica. Estos resultados indican que los niveles de energía en la pastura se encuentran por debajo de los mínimos requeridos (10 a $12 \mathrm{MJ}$ EM/kg MS) para llevar adelante una producción lechera de 5.3 1/vaca/día (Geenty y Rattray, 1987). Por otro lado, León et al. (2012) reportaron valores de $8.0 \mathrm{MJ} / \mathrm{kg}$
MS bajo riego. Se reconoce que pastos con valores superiores a $8.37 \mathrm{MJ} / \mathrm{kg}$ MS se les considera de buena calidad (Verdecia et al., 2008).

\section{Calcio}

El contenido de calcio en la dieta presentó diferencias significativas entre épocas $(p<0.01)$, oscilando entre $0.80,0.29$ y $0.14 \%$ en las épocas inicio de lluvias, lluviosa y seca, respectivamente (Figura 3). El mayor contenido de calcio al inicio de la época lluviosa se debió principalmente a la presencia de rebrotes tiernos y suaves y el menor valor se registró en la época seca $(0.14 \%)$. Se estima que los requerimientos de calcio para mantenimiento de las vacas variaron entre 27.8 , 28.5, y $61.3 \mathrm{~g} / \mathrm{vaca} /$ día (NRC, 2001) durante las épocas seca, inicio de lluvia y lluviosa, respectivamente; sin embargo, las vacas solo consumieron $1.62,11.89$ y $4.09 \mathrm{~g} / \mathrm{vaca} /$ día. Los resultados revelan que el forraje no cubre los requerimientos de calcio en las tres épocas, por lo que se debería complementar con otras fuentes. 
Cuadro 1. Variables utilizadas en el cálculo del perfil alimentario

\begin{tabular}{lcc}
\hline Indicador & Unidad & Cantidad \\
\hline Oferta de forraje (promedio periodo) & $\mathrm{kg} \mathrm{MS} / \mathrm{ha}$ & 5991 \\
Vacas en producción & - & 25 \\
Área de la Unidad Agropecuaria & $\mathrm{ha}$ & 41.9 \\
Peso vivo de las vacas & $\mathrm{kg}$ & 474 \\
Producción de leche & litros/periodo de 10 meses & 1682.7 \\
Grasa de leche & $\%$ & 2.87 \\
Sistema propuesto & $\mathrm{kg}$ de grasa de leche/vaca/año & 48.35 \\
\hline
\end{tabular}

Lamela et al. (1995) y Machado y Olivera (2004) reportan valores de calcio de 0.66 y $0.96 \%$, respectivamente, mientras que Verdecia (2008) reporta concentraciones de 0.48 y $0.35 \%$ para épocas poco lluviosa y lluviosa, respectivamente, siendo estos valores mayores a los encontrados en este estudio $(0.14$ y 0.28 en las épocas seca y lluviosa, respectivamente). Sin embargo, el promedio general en este estudio $(0.41 \%)$ estuvo por encima del $0.27 \%$ reportado por Homen et al. (2010) para la misma especie. Se resalta el hecho de que los valores promedio estuvieron por encima del nivel crítico $(0.17 \%)$ para el mantenimiento animal, más no para la producción de leche (Minson, 1981).

\section{Fósforo}

La cantidad de fósforo en el forraje fue de $0.16,0.19$ y $0.23 \%$ en las épocas seca, inicio de lluvias y lluviosa, respectivamente (Figura 3), sin diferencias significativas entre épocas. El promedio de fósforo en el forraje fue de $0.19 \%$; valor cercano al $0.23 \%$ reportado por Lamela et al. (1995) y muy cercanos al valor crítico $(0.19 \%)$, según la NRC (2001). Esto se debe que el inicio de los rebrotes coincide con la mayor precipitación pluvial. Similar tendencia fue encontrada por Homen et al. (2010) para la época de lluvia $(0.23 \%)$ y seca $(0.16 \%)$ y por Machado y
Olivera (2004) con 0.22 y 0.12 en las épocas de lluvia y poco lluviosa, respectivamente. Tejos y Rodríguez (1995) mencionan que este comportamiento se debe a que el agua constituye un vehículo natural para el movimiento de los iones del suelo, actuando en la absorción de este elemento y su disponibilidad en el suelo (Casanova y Eduardo, 2005).

Los requerimientos de fósforo fueron $20.6,21.1$ y $30.1 \mathrm{~g} / \mathrm{vaca} /$ día para las épocas seca, inicio de lluvia y lluviosa, respectivamente (NRC, 2001). Las vacas consumieron $1.9,2.8$ y $3.4 \mathrm{~g} / \mathrm{vaca} /$ día en cada época respectivamente. En consecuencia, el forraje no estaba cubriendo los requerimientos de fósforo de los animales. Es posible que sea debido a los niveles medios de fósforo encontrados en el suelo (9.00 ppm). La deficiencia de este elemento en el suelo provoca una marcada reducción en el crecimiento y calidad del forraje (Quintero y Boschetti, 2005). Los fertilizantes fosfatados representan una buena opción para incorporar fósforo a la pradera (Fixen, 2003).

\section{Perfil Alimentario}

Las variables utilizadas en el perfil alimentario se muestran en el Cuadro 1. El perfil determinó una carga de 1.5 vacas/ha con un balance positivo $(+1470.9 \mathrm{~kg} \mathrm{MS} / \mathrm{ha})$ 
Cuadro 2. Perfil alimentario para vacas Brown Swiss x criollas al pastoreo sobre una pastura de Paniucum maximum en Cajamarca, Perú

\begin{tabular}{lccc}
\hline Variable & Seca & $\begin{array}{c}\text { Inicio de } \\
\text { lluvia }\end{array}$ & Lluvia \\
\hline Producción de leche, kg/vaca/día & 6.80 & 4.94 & 4.16 \\
Producción de grasa de leche, kg/vaca/día & 0.20 & 0.13 & 0.13 \\
Requerimientos energéticos, MJ EM/vaca/día & 101.97 & 92.91 & 107.33 \\
VEF del forraje, MJ EM/Kg MS & 3.94 & 7.00 & 5.23 \\
Asignación de forraje, kg MS/vaca/día & 11.58 & 14.87 & 14.62 \\
Carga animal propuesta, vaca/ha & 1.5 & 1.5 & 1.5 \\
Demanda de hato lechero, kg MS/ha/día & 16.90 & 21.71 & 21.34 \\
Tasa de crecimiento de forraje, $\mathrm{kg} M S / \mathrm{ha} / \mathrm{día}$ & 8.16 & 18.42 & 46.28 \\
Cambio en disponibilidad de forraje, $\mathrm{kg} \mathrm{MS} / \mathrm{ha}$ & -1066.16 & -404.75 & 1470.91 \\
Disponibilidad al inicio de época, $\mathrm{kg} \mathrm{MS} / \mathrm{ha}$ & 2696.86 & 4023.29 & 7764.29 \\
Disponibilidad más crecimiento, $\mathrm{kg} \mathrm{MS} / \mathrm{ha}$ & 3692.73 & 6288.51 & 10494.55 \\
Disponibilidad al final de la época, $\mathrm{kg} \mathrm{MS} / \mathrm{ha}$ & 1630.69 & 3618.54 & 9235.20 \\
Utilización, $(\%)$ & 55.84 & 42.46 & 12.00 \\
\hline
\end{tabular}

entre la oferta y la demanda para la época lluviosa, debido a la mayor tasa de crecimiento del forraje (Cuadro 2). Para ello, se calculó los requerimientos de MS de forraje (4103.72 $\mathrm{kg} \mathrm{MS} / \mathrm{vaca} /$ periodo) entre la grasa de leche (48.35 kg de grasa/vaca/año), dando como resultado $84.88 \mathrm{~kg} \mathrm{MS} / \mathrm{kg}$ grasa de leche. Luego, la carga animal se calculó dividiendo la oferta de forraje $(5991.36 \mathrm{~kg} \mathrm{MS} / \mathrm{ha} / \mathrm{pe}-$ riodo) entre los requerimientos de MS de forraje (4103.72 kg MS/vaca/periodo) dando como resultado 1.5 vacas/ha.

La utilización de forraje fue de $55.84 \%$ para la época seca, debido a la poca disponibilidad y baja calidad del forraje, lo cual llega a cubrir los requerimientos energéticos de las vacas. En el caso de la época lluviosa se tuvo una menor de utilización de la pastura (12.0\%) debido a la mayor tasa de crecimiento. Las especies de Panicum pueden soportar pastoreos intensivos, siempre y cuando se mantenga una apropiada fertilidad y humedad del suelo, llegando a soportar hasta 4 animales/ha en época lluviosa y 2 animales/ha en época seca (CIAT, 2002). Sin embargo, en un sistema silvopastoril en secano, $P$. maximum puede soportar entre $2.0 \mathrm{UA} / \mathrm{ha} /$ año (González, 2013) y 2.5 UA/ha/año (Mármol, 2006).

La utilización promedio de forraje fue solo de $38.6 \%$ (Cuadro 2), resultando en una subutilización de la pastura. Ñaupari (2000) reportó, asimismo, entre 19 y $39 \%$ de uso de pasturas asociadas Lolium perenne y Trifolium repens con vacas lecheras en los meses lluviosos y poco lluviosos, lo cual indica que la pastura estaba siendo subutilizada. Machado (2013) indica que el Panicum maximum Jacq (accesión CIH-22), en pastoreos rotativos en suelos de mediana fertilidad, puede llegar a ser utilizado hasta $54.8 \%$ debido a la alta proporción de hojas (80.7\%). En el pastoreo de especies de Panicum se debe considerar un residual de $20 \mathrm{~cm}$ de altura como mínimo para asegurar el rebrote (CIAT, 2000). 


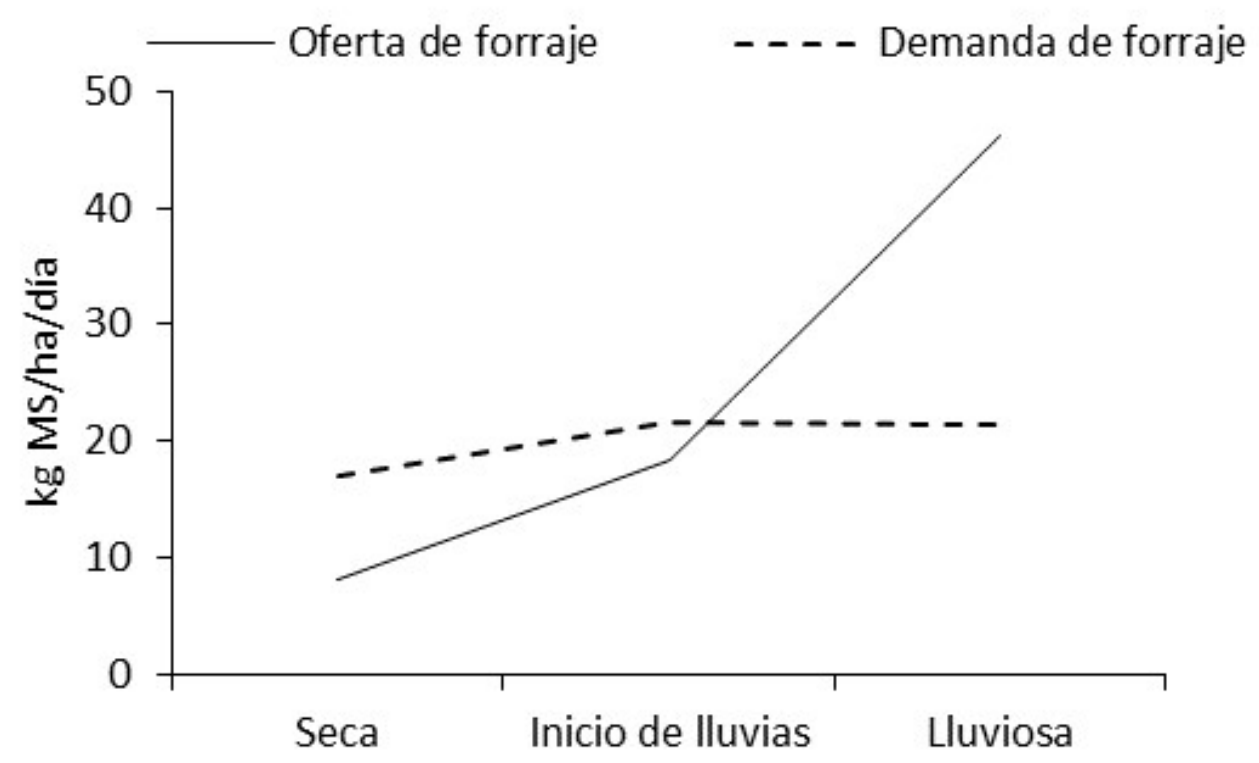

Figura 4. Balance entre oferta y demanda de forraje de Panicum máximum de vacas al pastoreo en Cajamarca, Perú

La unidad agropecuaria en estudio está subutilizando la pastura. Loayza (2008), al estudiar $P$. maximum bajo riego, con toretes y vaquillas con sistemas de pastoreo rotativo diarios, concluye que la mayor eficiencia de uso es de 87.7 y $87.0 \%$, respectivamente, ocasionado por una mayor presión de pastoreo que induce una disminución en la selectividad de los animales y un consumo más homogéneo. Se entiende por utilización eficiente de un forraje el alto grado de consumo (60$80 \%$ ), el cual es indirectamente proporcional a la disponibilidad de forraje (Formoso, 2005). El pastoreo eficiente, se basa en el uso de un alto número de animales por unidad de área, en potreros pequeños, con alta frecuencia de rotación y largos periodos de descanso (Anzola, 2014). Rodríguez et al. (1996) mencionan que la implementación de un sistema de pastoreo rotativo permite aumentar la cantidad de MS consumida por superficie; es decir, se utiliza más eficiente-mente el forraje mediante el manejo del tiempo de perma- nencia en cada potrero y el descanso. A medida que aumenta la carga por unidad de superficie disminuye la asignación diaria de forraje por animal y el consumo individual; sin embargo, para el mejor aprovechamiento de los forrajes, se debe establecer la carga animal, el intervalo y duración del pastoreo (Sánchez, 2007).

El perfil alimentario revela que existe un periodo deficitario de forraje para la época seca (Figura 4). Esta situación indica que se debe hacer un balance de la oferta y demanda dentro de los periodos de abundancia y deficiencia a fin de optimizar el recurso forrajero.

\section{Conclusiones}

- El valor energético y la disponibilidad del pasto mejora considerablemente en la época lluviosa, pero sin llegar a cubrir el 
nivel óptimo para producción de leche (10-12 MJ EM/kg MS).

- La carga actual de $1.5 \mathrm{UA} / \mathrm{ha} /$ año es menor a la capacidad de carga esperada de $3 \mathrm{UA} / \mathrm{ha} /$ año para una pastura de Panicum maximum, ocasionando una subutilización de la pastura y baja producción animal.

- Los niveles de calcio y fósforo no cubrieron las necesidades de los animales, lo cual se sugiere cubrir con otras fuentes.

\section{Literatura Citada}

1. Alais C. 1994. Ciencia de la leche. Principios de técnica lechera. Barcelona, España: Ed. Continental. 873 p.

2. Anzola H, Durán H, Rincón J, Martínez J, Restrepo J. 2014. El uso eficiente de los forrajes tropicales en la alimentación de los bovinos. Rev Cienc Anim 7: 111-132.

3. [AOAC] Association of Official Analytical Chemist. 2001. Official methods of analysis. 4.2.11 AOAC Official Method 2001.11. [Internet]. Disponible en: http://www.freedocumentsearch.com/pdf/aoac-officialmethod-976.06-rotein.html

4. [ASRM] American Society of Range Management and Agriculture Board. 1962. Basic problems and techniques in range research. Washington DC, USA: National Academy of Sciences Pub. $\mathrm{N}^{\mathrm{o}}$. $890.336 \mathrm{p}$

5. Baldelomar Z, Rojas C, Cortez M. 2004. Producción y análisis bromatológico de tres gramíneas tropicales $(B$. decumbens, Panicum maximum, $\mathrm{cv}$ Tanzania y cv Gatton). Tesis de Médico Veterinario Zootecnista. Santa Cruz, Bolivia: Univ. Autónoma Gabriél René Moreno. 74 p.

6. Barreira SR. 2008. Crecimiento del arbolado, producción de pasto y efectos edáficos en sistemas silvopastorales fertilizados con lodos de depuradora. Efecto residual. Tesis Doctoral. Galicia, España: Univ. Santiago de Compostela. 284 p.
7. Baruch Z, Fisher M. 1991. Factores climáticos de competencia que afectan el desarrollo de la planta en el crecimiento. En: Establecimiento y renovación de pasturas. Conceptos, experiencia y enfoques de la investigación. Cali, Colombia: Red de Investigación y Evaluación de Pastos Tropicales - CIAT. 103 p.

8. Bernal J. Espinosa J. 2003. Manual de nutrición y fertilización de pastos. Canadá: IPNI. $94 \mathrm{p}$

9. Casanova O, Eduardo F. 2005. Introducción a la ciencia del suelo. Caracas: UCV Consejo de Desarrollo Científico y Humanístico. $482 \mathrm{p}$.

10. Cerdas R, Vallejos E. 2011. Disponibilidad de biomasa del pasto guinea (Megathyrsus maximus) Tanzania con varias fuentes y dosis de nitrógeno en Guanacaste, Costa Rica. InterSedes 12(23). [Internet]. Disponible en: https:/ /revistas.ucr.ac.cr/index.php/intersedes/ article/view/975/1036

11. Cerdas R. 2011. Programa de fertilización de forrajes. Desarrollo de un módulo práctico para técnicos y estudiantes de ganadería de Guanacaste, Costa Rica. InterSedes 12(24). [Internet]. Disponible en: https://revistas.ucr.ac.cr/ index.php/intersedes/article/view/967/ 1028

12. Decreto Supremo $N^{\circ}$ 017-2009-AG. 2009. Reglamento de clasificación de tierras por su capacidad de uso mayor. 2 de setiembre del 2009. Diario Oficial El Peruano.

13. Fixen P. 2003. Dinámica del fósforo en el suelo y en el cultivo en relación al manejo de los fertilizantes fosfatados. Informaciones Agronómicas 16: 3-5.

14. [CIAT] Centro de Investigación de Agricultura Tropical. 2002. Tropical grasses and legumes: optimizing genetic diversity for multipurpose use. Anual Report. Project IP-05. Cali, Colombia: CIAT. 289 p.

15. [CIAT] Centro de Investigación de Agricultura Tropical. 2000. Tropical grasses and legumes: optimizing genetic diversity for multipurpose use. Anual 
Report. Project IP-05. Cali, Colombia: CIAT. 215 p.

16. Zaragoza J, Hernández A, Pérez J, Herrera J, Osnaya F, Martínez P, González S, et al. 2009. Análisis de crecimiento estacional de una pradera asociada alfalfa-pasto ovillo. Tec Pecu Méx 47: 173-178.

17. Derner J, Hunt L, Filho K, Ritten J, Capper J, Han G. 2017. Livestock production systems. In: Briske DD (ed), Rangeland systems. Processes, management and challenges. Springer. p 347-372.

18. Flores E. 1993. Applying the concept of feeding stations to the behavior of cattle grazing variable amounts of available forage. MSc Thesis. USA: Utah State University. 58 p.

19. Formoso F. 2005. Eficiencia de la producción y utilización de forraje en otoño e invierno. Jornada Producción Animal Intensiva. Uruguay. INIA.

20. Geenty G, Rattray, PV. 1987. The energy requirements of grazing sheep and cattle. In: Livestock feeding on pasture. New Zealand Soc Anim Prod 10. 39-53.

21. González JM. 2013. Costos y beneficios de un sistema silvopastoril intensivo (SSPi), con base en Leucaena leucocephala (estudio de caso en el municipio de Tepalcatepec, Michoacán, México). Avances Invest Agropec 17: 35-50.

22. Harper K, McNeill D. 2015. The role iNDF in the regulation of feed intake and the importance of its assessment in subtropical ruminant systems (the role of iNDF in the regulation of forage intake). Agriculture 5: 778-790. doi: 10.3390/ agriculture 5030778

23. Holdridge L. 2000. Ecología basada en zonas de vida. San José, Costa Rica: IICA. $216 \mathrm{p}$.

24. Homen M, Entrena I, Arriojas L, Ramia M. 2010. Biomasa y valor nutritivo del pasto Guinea Megathyrsus maximus (Jacq.) B.K. Simon \& S.W.L. Jacobs. «Gamelote» en diferentes perio- dos del año en la zona de bosque húmedo tropical, Barlovento, estado Miranda. Zootec Trop 28: 255-265.

25. [INEI] Instituto Nacional de Estadística e Informática. 2012. IV Censo Nacional Agropecuario. Resultados finales. INEI Lima Perú. [Internet]. Disponible en: https://proyectos.inei.gob.pe/ CenagroWeb/

26. Lamela L, Fung C, Esparza R. 1995. Comportamiento del Panicum maximum cv. SIH 127 para la producción de leche. Pastos y Forrajes 18: 263-269.

27. León M, Martínez SJ, Pedraza RM, González, C, Barreto G. 2012. Correlación entre energía metabolizable calculada y producción de gas in vitro con heces depuestas como inóculo. Rev Prod Anim 24(2).

28. Loayza J. 2008. Evaluación del pasto Saboya (Panicum maximum Jacq) en el periodo de mínima precipitación, sometido a tres sistemas de pastoreo, en el acabado de toretes y vaconas Charbray, en la hacienda San Antonio. Tesis de Ingeniero Agropecuario. Ecuador: Escuela Politécnica del Ejército. $60 \mathrm{p}$.

29. Long S, Zhu X, Naidu S, Ort D. 2006. Can improvement in photosynthesis increase crop yields? Plant Cell Environ 29: 315-330.

30. López G, Nuñez J, Aguirre L, Flores E. 2018. Dinámica de la producción primaria y valor nutritivo de tres gramíneas tropicales (Melinis minutiflora, Setaria sphacelata y Brachiaria mutica) en tres estados fenológicos. Rev Inv Vet Perú 29: 396-409. doi: 10.15381/ rivep.v29i2.14494

31. Machado R. 2013. Comportamiento de 19 accesiones de Panicum maximum Jacq bajo condiciones de pastoreo en un suelo de mediana fertilidad. Pastos y Forrajes 36: 202-208.

32. Machado R, Olivera Y. 2004. Evaluación de genotipos mejorados de Panicum maximum en condiciones de pastoreo simulado y sombra. Pastos y Forrajes 24: 117. 
33. [MAFF] Ministry of Agriculture, Fisheries and Food. 1975. Energy allowances and feeding systems for ruminants. UK: MAFF, Technical Bulletin $33.79 \mathrm{p}$.

34. Mármol JF. 2006. Manejo de pastos y forrajes en la ganadería de doble propósito. En: X Seminario de Pastos y Forrajes. Maracay, Venezuela: Univ. de Zulia.

35. Martínez M. 1971. Calibración y comparación de algunos métodos para la determinación de humedad del suelo. Tesis de Maestría. Chapingo, México: Colegio de Postgraduados. $120 \mathrm{p}$.

36. Milligan K, Brookes M, Thomson $K$. 1987. Feed planning on pasture. In: Livestock feeding in pasture. New Zealand Soc Anim Prod 10: 75-88.

37. Minson D. 1981. Nutritional differences between tropical and temperate pastures. In: Morley FHM (ed). Grazing animals. Amsterdam: Elsevier Scientific. p 143-157.

38. Molano M. 2012. Caracterización nutricional de forrajes tropicales usando espectroscopía de infrarrojo cercano (NIRS). Tesis Doctoral. Palmira: Univ. Nacional de Colombia. $168 \mathrm{p}$.

39. [NRC] National Research Council. 2001. Nutrient requirements of dairy cattle. $7^{\text {th }}$ rev. ed. Washington DC, USA: National Academic Press. 408 p.

40. Naupari J. 2000. Comportamiento nutricional y perfil alimentario de vacas lecheras en pastos cultivados Rye grass/ trébol de la U.P. Consac. Tesis de Magister. Lima: Univ. Nacional Agraria La Molina. $87 \mathrm{p}$.

41. Naupari J, Flores E. 2002. Comportamiento nutricional y perfil alimentario de vacas lecheras en pastos cultivados ryegrass/trébol de la U.P. Consac. Rev Anales Cient UNALM 1:21-36.

42. Peng S, Huang J, Sheehy JE, Laza $R C$, Visperas RM, Zhong $X$, Centeno GS, et al. 2004. Rice yields decline with higher night temperature from global warming. P Natl Acad Sci USA 101: 9971-9975. doi: 10.1073/pnas.0403720101.
43. Pentón G 2000. Efecto de la sombra de los arboles sobre el pastizal en un sistema seminatural. Tesis de Maestría. Nicaragua: Univ. de Matanzas.

44. Quintero C, Boschetti N. 2005. Manejo del fósforo en pasturas. Instituto Nacional de Tecnología Agropecuaria. Argentina. [Internet]. Disponible en: http:// www.infogranjas.com.ar/agricultura/ 310-pasturas-ferilizacion/1342-manejodel-fosforo-en-pasturas-

45. Ramírez O, Hernández A, Carneiro $S$, Pérez J, Francisco J, Raymuno A, Guadalupe J, et al. 2009. Acumulación de forraje, crecimiento y características estructurales del pasto Mombaza (Panicum maximum Jacq.) cosechado a diferentes intervalos de corte. Tec Pecu Mex 47: 203-213.

46. Rodríguez M, Sanabria D, Manrique U, Fariñas J, Rivas E. 1996. Efecto de cinco niveles de nitrógeno sobre el comportamiento de Digitaría swazilandensis Stent en el campo experimental Santa Bárbara, MonagasZootec Trop 14: 133-147.

47. Romero G 2018. Estado nutricional del pasto guinea (Panicum maximun jacq) asociado con faique (Acacia macracantha) en un sistema silvopastoril en Jaén - Cajamarca. Tesis de Magíster. Lima: Univ Nacional Agraria La Molina. 69 p.

48. Ruiz F, Rodríguez E, Pinzón J, Anzola H, y Castro L. 2015. Establecimiento y evaluación del guinea Panicum maximum cv. Massai en la hacienda Guachicono del Bordo, Patía (Cauca). Rev Cienc Anim 9: 125-154.

49. Sánchez J. 2007. Utilización eficiente de las pasturas tropicales en la alimentación del ganado lechero. En: XI Seminario de Pastos y Forrajes en Sistemas de Producción Animal. Barquisimeto, Venezuela.

50. Snider JL, Oosterhuis DM, Skulman BW, Kawakami EM. 2009. Heat stress-induced limitations to reproductive success in Gossypium hirsutum. Physiol Plantarum 137: 125-138. doi: 10.1111/ j.1399-3054.2009.01266.x 
51. Tejos M, Rodríguez C. 1995. Adaptación de nuevas gramíneas al llano bajo venezolano. Rev Fac Agron Luz 15: 278-282.

52. Tilley M, Terry A. 1963. Two stage technique for the in vitro digestion of forage crops. J Brit Grassland Soc 18: 104-111. doi: 10.1111/j.13652494.1963.tb00335.x

53. Verdecia DM. 2008. Rendimiento y componentes del valor nutritivo del Panicum maximum cv. Tanzania. REDVET 9(5). [Internet]. Disponible en: http://www.redalyc.org/articulo.oa $? \mathrm{id}=63611397008$

54. Verdecia M, Ramírez J, Leonard I, García F. 2008. Potencialidades agro- productivas dedos cultivares de Panicum maximum (c.v Mombasa y Uganda) en la provincia Granma. REDVET 10(5). [Internet]. Disponible en: http://www.veterinaria.org/revistas/ redvet/n050509/050901.pdf

55. Vidal I. 2008. Relación entre el consumo aparente de forraje en primavera y la producción de leche de vacas en pastoreo. Tesis de Ingeniero Agrónomo. Temuco, Chile: Univ. de la Frontera. 86 p.

56. Wahid A, Gelani S, Ashraf M, Foolad MR. 2007. Heat tolerance in plants: an overview. Environ Exp Bot 61: 199-223. doi: 10.1016/j.envexpbot.2007.05.011 